\title{
Effect of Repetitive Transcranial Magnetic Stimulation on Depression and Cognition in Individuals With Traumatic Brain Injury: A Meta- Analysis
}

\section{Ping-Yen Tsai}

School of Medicine, College of Medicine, Taipei Medical University, Taipei

\section{Yang-Ching Chen}

Department of Family Medicine, School of Medicine, College of Medicine, Taipei Medical University,

Taipei

\section{Jia-Yi Wang}

Department of Neurosurgery, School of Medicine, College of Medicine, Taipei Medical University, Taipei

\section{Kuo-Hsuan Chung}

Department of Psychiatry, School of Medicine, College of Medicine, Taipei Medical University, Taipei

Chien-Hung Lai ( $\square$ chlai@tmu.edu.tw )

Department of Physical Medicine and Rehabilitation, School of Medicine, College of Medicine, Taipei Medical University, Taipei

\section{Research Article}

Keywords: rTMS, TBI, SMD, DLPFC, Transcranial Magnetic

Posted Date: March 15th, 2021

DOI: https://doi.org/10.21203/rs.3.rs-285574/v1

License: (c) (i) This work is licensed under a Creative Commons Attribution 4.0 International License. Read Full License

Version of Record: A version of this preprint was published at Scientific Reports on August 20th, 2021. See the published version at https://doi.org/10.1038/s41598-021-95838-2. 


\section{Effect of Repetitive Transcranial Magnetic Stimulation on Depression and}

\section{Cognition in Individuals With Traumatic Brain Injury: A Meta-Analysis}

Running title: Repetitive Transcranial Magnetic Stimulation on Depression in Traumatic Brain Injury

Ping-Yen Tsai, M.D., M.S. ${ }^{1}$; Yang-Ching Chen, M.D., Ph.D. ${ }^{2,3}$; Jia-Yi Wang, Ph.D. ${ }^{4,5}$; Kuo-Hsuan Chung, M.D. ${ }^{6,7}$; Chien-Hung Lai, M.D., Ph.D. ${ }^{8,9^{*}}$

1. School of Medicine, College of Medicine, Taipei Medical University, Taipei, Taiwan

2. Department of Family Medicine, School of Medicine, College of Medicine, Taipei Medical University, Taipei, Taiwan

3. Department of Family Medicine, Taipei Medical University Hospital, Taipei, Taiwan

4. Department of Neurosurgery, School of Medicine, College of Medicine, Taipei Medical University, Taipei, Taiwan

5. Neuroscience Research Center, Taipei Medical University, Taipei, Taiwan

6. Department of Psychiatry, School of Medicine, College of Medicine, Taipei 
Medical University, Taipei, Taiwan

7. Department of Psychiatry, Taipei Medical University Hospital, Taipei, Taiwan

8. Department of Physical Medicine and Rehabilitation, School of Medicine, College of Medicine, Taipei Medical University, Taipei, Taiwan

9. Department of Physical Medicine and Rehabilitation, Taipei Medical University Hospital, Taipei, Taiwan

\section{E-mail addresses of all authors}

Ping-Yen Tsai: b101105135@tmu.edu.tw

Yang-Ching Chen: melisa26@tmu.edu.tw

Jia-Yi Wang: jywang2010@tmu.edu.tw

Kuo-Hsuan Chung: ch2006ung@tmu.edu.tw

Chien-Hung Lai: chlai@tmu.edu.tw

*Corresponding author:

Chien-Hung Lai MD, PhD, Associate Professor [ORCID ID: https://orcid.org/0000$\underline{0002-5765-3120]}$,

Department of Physical Medicine and Rehabilitation, Taipei Medical University Hospital, No. 252, Wu-Hsing St., Taipei City 110, Taiwan

Tel: 886-2-27372181 ext. 3538 ; Fax: 886-2-55589880

E-mail: chlai@tmu.edu.tw 


\section{Abstract}

Repetitive transcranial magnetic stimulation (rTMS) is an FDA-approved therapy in major depressive disorder. However, its treatment efficacy on depression after traumatic brain injury (TBI) remains inconclusive. We conducted a meta-analysis to assess the effectiveness of executing rTMS over dorsolateral prefrontal cortex (DLPFC) on depression, cognitive impairment and post-concussion syndrome in individuals with traumatic brain injury. This study contained seven randomized controlled trials that published before April 5, 2020 in PubMed, Embase, Scopus, Cochrane, and Web of Science databases. The rTMS had significant anti-depressant effect ( $\mathrm{SMD}=1.03)$, but the effects dissipated at one-month follow-up (SMD = 0.39). In the subgroup analysis, only applying rTMS to left DLPFC area of post-TBI patients showed significant anti-depressant effect $(\mathrm{SMD}=0.98)$. Moreover, current data observed that rTMS on post-TBI patients possessed substantial improvement in visuospatial memory $(\mathrm{SMD}=0.39)$, but wasn't in processing speed $(\mathrm{SMD}=-0.18)$ and selective attention $(\mathrm{SMD}=0.21)$. In addition, the effect of rTMS on postconcussion syndrome was insignificant. In conclusion, the short-term antidepressant effect of left DLPFC rTMS in patients with TBI was significant. Furthermore, the effectiveness of rTMS on cognition and post-concussion syndrome in patients with post-TBI depression was limited. 


\section{Introduction}

Traumatic brain injury (TBI) has been recognized as a global health concern in recent years. Every year, 27-69 million people worldwide experience TBI ${ }^{1,2}$. TBI not only is a major cause of death and disability but also leads to many neurological and psychological sequelae that increase global burden, including depression and cognitive impairment ${ }^{3,4}$. Within the first year after TBI, the prevalence rate of major depressive disorder ranges from $25 \%$ to $53 \%{ }^{5,6}$. From 2007 to 2017 , depressive disorder was the third leading burden-causing disease ${ }^{7}$. Cognitive impairment, characterized by alterations in judgment, memory, attention, planning, processing speed, and executive functions, is another complication that limits daily activities following TBI ${ }^{8}$. Approximately $33 \%$ of patients with mild TBI have experienced short-term functional impairment, $80 \%$ of which has resolved within 6 months ${ }^{9}$. Moderate to severe TBI may lead to persistent impairment in cognitive function at 6 months ${ }^{10}$. Although one-third of patients with severe TBI have normal neuropsychological test performance at 3 months, patients with TBI with cognitive impairment have high future disability risk ${ }^{10}$. Therefore, early therapeutic intervention for post-TBI depression and cognitive impairment has become increasingly critical. 
Functional neuroanatomy provides insight into the pathomechanism of depression and cognitive impairment. Left dorsolateral prefrontal cortex (DLPFC) hypoactivity and right DLPFC hyperactivity are involved in depression pathogenesis. Depression severity is associated with right DLPFC hyperactivity ${ }^{11}$. The DLPFC is responsible for emotional judgement as well as cognitive and executive function ${ }^{12}$. The functional connections between the prefrontal cortex and other regions of the brain form different networks, including the default mode network and cognitive control network ${ }^{13,14}$. TBI leads to diffuse axonal injury that disrupts network synchrony ${ }^{15}$. To restore functional connectivity, a functional magnetic resonance imaging-based approach was used to target and modulate specific cortical areas in TBI-induced depression ${ }^{16}$.

Repetitive transcranial magnetic stimulation (rTMS) is a noninvasive neuromodulation approach; in rTMS, electric current is targeted at a specific area by using time-dependent magnetic fields. Studies have demonstrated that a high frequency of rTMS facilitates neuronal activity, whereas a low frequency suppresses neuronal excitement. Neuronal activity can be modulated through pulse frequency adjustment ${ }^{17}$. For clinical application, rTMS has been proved effective in improving symptoms of several diseases, including Parkinson disease ${ }^{18}$, Alzheimer disease ${ }^{19}$, 
stroke $^{20}$, and treatment-resistant depression (TRD) ${ }^{21}$. Thus, rTMS has been proposed for treating people with TBI. Nevertheless, the effect of rTMS on TBI-induced depression or cognitive impairment remains controversial, although related publications have increased from 2018 to 2020. Thus, a systematic review with metaanalysis is crucial to validate the effectiveness of rTMS in treating post-TBI complications. A meta-analysis by Gao et al. revealed that high-frequency rTMS (HF rTMS) over the left DLPFC and low-frequency rTMS over the right DLPFC exhibited similar effects on major depressive disorder ${ }^{22}$. However, to our knowledge, no systematic review with meta-analysis has been conducted to ascertain the effectiveness of rTMS on TBI-induced depression or cognitive impairment. Therefore, the principle aim of this meta-analysis is to determine the efficacy of rTMS over DLPFC in treating TBI-induced depression. Next, our secondary purpose is to investigate whether rTMS can improve cognitive decline and post-concussion syndrome in patients with depression. Final, current study also evaluates the stimulation parameters of rTMS and the quality of the past studies to make suggestions for future researches. 


\section{Results}

\section{Study selection}

Through the initial database search, 1467 articles were identified. After removing duplicate studies, 876 articles were obtained, and after further review of titles and abstracts, we obtained 65 articles. The 65 studies were thoroughly reviewed, and most of them were excluded due to their non-RCT design and other outcomes. Finally, seven RCTs that compared the effects of rTMS treatment with sham on post-TBI depression, cognitive impairment, or postconcussion syndrome (PCS) were included (Figure 1a).

\section{Study characteristics}

Table 1 presents the characteristics of the selected studies. In total, 136 participants were included in our research; 79 participants who had experienced TBI and had post-TBI depression were included in five studies; 57 participants with a diagnosis of posttraumatic headache or PCS had moderate to severe depression on average in two studies. The participants in the included articles had a mean age of $34.1-46.3$ years and a higher percentage of men $(73 ; 54 \%)$ than women. The rTMS protocols were summarized in Table 2. 


\section{Measured outcomes}

Depression

Four studies reported depression severity according to the MADRS ${ }^{16,23-25}$, and two articles used the HAMD to assess depression ${ }^{26,27}$. Another study by Stilling et al. measured the degree of depression by using the Patient Health Questionnaire 9 (PHQ9) ${ }^{28}$. By using the mean values of baseline depression severity score, six studies [Hoy et al. (MADRS $=34.0)$, Siddiqi et al. (MADRS $=32.2)$, Lee et al. (MADRS $=23.8)$, Rao et al. $(\mathrm{HAMD}=23.5)$, Leung et al. $(\mathrm{HAMD}=23.9)$, and Stilling $(\mathrm{PHQ}-9=$ 11.9)] enrolled participants with moderate to severe depression (MADRS $>19$, HAMD $>13$, and PHQ-9 > 9) ${ }^{16,24-28}$. A study by Moussavi et al. recruited participants with light PCS (LPCS) and severe PCS (SPCS). Participants with LPCS and SPCS had mild depression $(\mathrm{MADRS}=10.2)$ and moderate to severe depression $(\mathrm{MADRS}=$ 19.65), respectively ${ }^{23}$. Given the inclusion criteria of the present study, only participants with moderate to severe depression were included.

\section{Cognition and attention}

Three studies used the Trail Making Test (TMT), a neuropsychological test of processing speed, attention, and task shifting to examine cognitive function. The TMT can be further divided into TMT-A and TMT-B. In TMT-A, participants must connect 
25 circles numbered 1-25 in numerical order. In TMT-B, participants must alternate between numbers (1-13) and letters (A to L) in an ascending order (1-A-2-B-3-...) ${ }^{29}$. Two studies examined cognitive function by using TMT-A and TMT-B ${ }^{24,26}$, whereas another study by Lee et al. evaluated the outcome by summing the time required for TMT-A and TMT-B ${ }^{25}$.

The Stroop Color Word Test (SCWT) is a test of selective attention. A conflict is created through presenting a mismatch between the name of a color ("green") and the color of the printed word (e.g., the word green printed using red ink) ${ }^{30}$. To examine the ability to overcome the cognitive interference, the time required for the SCWT was measured in three studies ${ }^{24-26}$. In the Brief Visuospatial Memory Test (BVMT), the participants were asked to memorize and reproduce six types of shapes ${ }^{31}$. After three learning trials, the scores of all rounds were summed $(\max =2 \times 6 \times 3=36$; 2 points for each figure in three trials). Two articles used the BVMT ${ }^{24,26}$.

\section{Postconcussion syndrome}

Three studies recruited patients with PCS evaluated by using the self-reported Rivermead Post-Concussion Symptom Questionnaire (RPQ-3 and RPQ-13) ${ }^{23,26,28}$.

The RPQ-3 inquires into the symptoms of headache, nausea/vomiting, and dizziness, three early postconcussion symptoms. The RPQ-13 questionnaire inquires into 13 
self-reported conditions, namely noise sensitivity, sleep disturbance, fatigue, being irritable, feeling depressed, feeling frustrated, forgetfulness, poor concentration, taking longer to think, blurred vision, light sensitivity, double vision, and restlessness, to measure cognitive and emotional symptoms ${ }^{32}$.

\section{Study quality}

The baseline imbalance of patient characteristics in three articles ${ }^{16,24,27}$ and unclear allocation concealment in Rao et al. resulted in a risk of uncertainty bias in the randomization process. Because depression improvement could confound the measurement of somatic symptoms (i.e., PCS), such deviations may have increased bias risk in outcome assessment in two studies ${ }^{23,28}$. Four articles had risk of bias due to high drop-out or loss-to-follow-up rates $(>5 \%)^{16,23,24,26}$. The study by Lee et al. had a high risk of bias of outcome measurement owing to unblinded assessors ${ }^{25}$. Trials that were not analyzed according to prespecified protocols in four studies (Rao et al., Siddiqi et al., Lee et al., and Leung et al.) led us to identify a potential risk of reporting bias ${ }^{16,25-27}$ (Figure $1 b$ ).

\section{Effectiveness}

Depression 
Five studies evaluated the effect of rTMS treatment on depression and measured depression outcome immediately after rTMS treatment ${ }^{16,23-25,27}$. In a random-effects model immediately after rTMS intervention, rTMS had a high mean effect size of $1.03(95 \%$ confidence interval $[\mathrm{CI}]=0.20-1.86)$ without heterogeneity $\left(\mathrm{I}^{2}=0 \%\right.$; Figure 2a). However, 1 month after rTMS treatment, the effect size of rTMS became smaller and statistically nonsignificant (effect size $=0.39 ; 95 \% \mathrm{CI}=-1.15$ to $1.93 ; \mathrm{I}^{2}$ $=0 \%$, although only three studies were included in the analysis ${ }^{23,26,28}$ (Figure 2b).

In the subgroup analysis, we observed that the results of bilateral sequential DLPFC rTMS therapy had an effect size of 0.99 in treating post-TBI depression, but these results failed to reach statistical significance when compared with the sham group (95\% CI $=-1.54$ to $3.52 ; \mathrm{I}^{2}=0 \%$; Figure $2 \mathrm{c}$ ). By contrast, left DLPFC rTMS treatment of post-TBI depression exhibited significant effectiveness compared with the sham group (effect size $=0.98 ; 95 \% \mathrm{CI}=0.04$ to $1.92 ; \mathrm{I}^{2}=0 \%$ ) despite having an effect size similar to bilateral sequential DLPFC rTMS (Figure 2d). Only one study investigated the efficacy of right DLPFC rTMS intervention in post-TBI depression. Their data revealed that right DLPFC rTMS had a large effect size (1.44) after right DLPFC rTMS treatment although it did not reach statistical significance $(\mathrm{CI}=-1.03$ to $3.91 ; \mathrm{I}^{2}=0 \%$; Figure $2 \mathrm{e}$ ). 


\section{Cognition and attention}

The mean effect sizes of rTMS on processing speed were $-0.25(\mathrm{CI}=-0.62$ to $\left.0.12 ; \mathrm{I}^{2}=0 \%\right)$ for TMT-A and $-0.26\left(\mathrm{CI}=-1.70\right.$ to $\left.1.18 ; \mathrm{I}^{2}=0 \%\right)$ for TMT-B. When both TMT-A and TMT-B were synthesized, the effect size was -0.18 (CI $=-0.53$ to $0.17 ; \mathrm{I}^{2}=0 \%$ ). All the results favored the sham group but were not statistically significant (Figure 3a-c).

Three studies assessed the effectiveness of rTMS treatment in improving selective attention by using the SCWT. The effect size after rTMS intervention was small (effect size $=0.21 ; 95 \% \mathrm{CI}=-0.20$ to $0.62 ; \mathrm{I}^{2}=0 \%$; Figure $3 \mathrm{~d}$ ). These results indicated that the efficacy of rTMS therapy in restoring selective attention was limited. However, rTMS significantly improved visuospatial memory, with an effect size of $0.39\left(\mathrm{CI}=0.21\right.$ to $0.56 ; \mathrm{I}^{2}=0 \%$; Figure $\left.3 \mathrm{e}\right)$.

\section{Postconcussion syndrome}

The effect of rTMS on PCS was small (effect size $=0.34 ; 95 \% \mathrm{CI}=-0.34$ to $1.02 ; \mathrm{I}^{2}=0 \%$ ) based on RPQ-3 assessment. Moreover, the rTMS group did not exhibit a favorable outcome relative to the control group (effect size $=-0.5 ; 95 \% \mathrm{CI}=-0.97$ to $-0.04 ; \mathrm{I}^{2}=0 \%$ ) when RPQ-13 was used to examine the effectiveness of rTMS intervention (Figure 4a, b). 


\section{Discussion}

Several studies have attempted to use rTMS to enhance the recovery of individuals with post-TBI depression. However, insufficient evidence was discerned regarding the effect of TMS on people with post-TBI depression. Hence, this study was designed to investigate the effectiveness of rTMS in treating post-TBI depression. Our results demonstrated that rTMS has a significant on post-TBI depression immediately after administration. Nevertheless, the effect size decreased over time. We observed no significant effect 1 month after rTMS treatment compared with the sham group. However, sample sizes of previous studies were small. In addition, few studies have performed a follow-up assessment, making it difficult to determine longterm efficacy (e.g., 4 weeks) of rTMS intervention in the present study. Therefore, recruiting a sufficient number of participants and having a long follow-up period in future studies is essential.

Relevant studies have proved that both unilateral and bilateral rTMS are effective in individuals with depression. Moreover, their results did not conclude that bilateral rTMS was superior to unilateral stimulation in remitting TRD ${ }^{33}$. A study by Trevizol et al. revealed that bilateral rTMS was superior to unilateral in elderly individuals with TRD ${ }^{34}$. Subgroup analysis in the present study indicated that both 
left and bilateral rTMS had a large effect size, although the effect of bilateral rTMS did not reach statistical significance. Notably, however, Siddiqi et al. ${ }^{16}$ determined that bilateral rTMS had a large effect size on TRD in patients with TBI. Thus, their data indicated that bilateral rTMS could play a promising role in treating depression in people with TBI.

A systematic review by Martin et al. revealed that applying rTMS to the prefrontal cortex of patients with depression modestly enhanced the cognition of these patients. Furthermore, such an effect was independent of the antidepressant effect of rTMS ${ }^{35}$. However, the studies included in the present study did not indicate that rTMS therapy significantly contributed to improvement in cognition and PCS in patients with TBI. A previous non-RCT research, which enrolled patients with PCS (60\% having depression), demonstrated significant improvement in SCWT and PCS scores but not for TMT scores ${ }^{36}$. Our analysis indicated that brief visuospatial memory (BVMT) but not processing speed (TMT) or PCS improved after rTMS treatment. The rTMS treatment for the inhibitory ability of cognitive interference (SCWT) was borderline effective. In a systemic review evaluating the cognitive effect of HF rTMS on depression in patients, few studies in their review demonstrated significant cognition-enhancing effects of rTMS, including selection attention ( 3 of 
9), spatial memory ( 1 of 6 ), and processing speed (4 of 13) ${ }^{37}$. Moreover, another research demonstrated a modest effect of rTMS on cognition in patients with depression and a significant improvement only in TMT-A and TMT-B ${ }^{35}$. The present results differ from those of previous investigations on the effect of rTMS on cognition and PCS. One of the reasons for this may be that previous studies and our study recruited different populations. Furthermore, the parameters of rTMS treatment and the testing of outcome variables were different among previous studies and ours. However, further evaluation of the effectiveness of rTMS on cognition and PCS in patients with TBI is necessary. In addition, cognitive function consists of several domains that involve different cortex areas in the cognitive control network ${ }^{13}$; the pathophysiology of cognitive enhancement by applying rTMS to patients with TBI should be further investigated.

Establishing and developing a universal protocol of rTMS administration (for example, with respect to duration, number of sessions, and dosage of rTMS application) is essential for improving the depression status and cognitive function of individuals with TBI. A minimum of 4-week (20 sessions) rTMS treatment was needed for cognitive enhancement in patients with psychiatric problems ${ }^{38}$. Moreover, a randomized controlled study revealed that 8 weeks are required for rTMS to 
improve cognitive function, including memory, attention, visuospatial function, and language in patients with schizophrenia ${ }^{39}$. Teng et al. suggested that HF rTMS with 1200-1500 pulses/day with 20 sessions exerted the best antidepressant effect ${ }^{40}$, and another study found that cumulative sessions rather than pulses determined the recovery rate in major depression ${ }^{41}$. The present meta-analysis demonstrated that previous studies used heterogeneous protocols, including number of sessions (4-20 times), total number of pulses (600-4000 times/day), application frequency, and stimulation intensity. Yet, anatomic variation between subjects may also have contributed to the heterogeneity of the results. TBI complications vary from patient to patient due to the highly heterogenous nature of the disease with various injury mechanisms ${ }^{42}$. This variability may result from the disruption of different brain networks underlying the mechanism of post-TBI complications. Therefore, stimulation protocols—-for example, location, frequency and intensity of stimulation, total number of pulses, as well as total amount of sessions — must be further determined.

This meta-analysis has several limitations. First, most of the included studies had a small sample size. Small sample size leads to low statistical power and subsequently limits the reliability of the results regarding the therapeutic effectiveness of rTMS in 
treating depression and cognitive impairment in patients following TBI. Second, although all the studies had applied rTMS to the left DLPFC area of the brain, stimulation protocols, including rTMS intervention duration, number of sessions, number of pulses, and stimulation intensity, varied. Thus, efficacy assessment and reproducibility of their investigations are difficult. Third, as few studies have executed a 1-month follow-up, determining the long-term effect of rTMS on depression and cognition of patients with TBI is difficult. Fourth, due to a lack of a mixed model design, determining the effect of rTMS on cognitive impairment is difficult in patients with TBI with concomitant depression status. Finally, several studies found variation in network topography between individuals, and image- or navigation-guided rTMS therapy may be considered in future work.

\section{Conclusion}

Our study demonstrated that rTMS has a short-term effect on post-TBI depression, whereas the effects of rTMS on cognitive impairment and PCS in patients with TBI remained inconclusive. In addition, RCT studies revealed that rTMS has a significant antidepressant effect only when applied to the left DLPFC area. However, previous studies were limited by small sample sizes and heterogenous methodologies. Further large sample sizes and well-designed trials are necessary to confirm the 
antidepressant and cognition-enhancing effects of rTMS and to establish optimal stimulation protocols for administering rTMS to patients with post-TBI depression.

\section{Methods}

\section{Literature search}

Multiple databases, including PubMed, Embase, Scopus, Cochrane, and Web of Science, were searched for randomized controlled trials (RCTs) published before April 5, 2020. The search terms were "traumatic brain injury [mesh]" AND "transcranial magnetic stimulation [mesh]." The meta-analysis was registered as CRD42020200348 in Prospero.

\section{Study selection}

Two authors independently screened the titles and abstracts of articles. Then, a full-text review was performed and eligible studies were included. In the case of discrepancy between two reviewers, the conflict was resolved through discussion among the two reviewers and a third reviewer to reach consensus. Studies fulfilling the following criteria were included: (1) patient with TBI, (2) patient with post-TBI depression (Montgomery-Asberg Depression Rating Scale [MADRS] $>6$ or Hamilton Rating Scale for Depression $[\mathrm{HAMD}]>7$ ) or participants having moderate 
to severe depression on average in the selected study, (3) age $\geq 18$ years, (4) the use of rTMS as intervention (or combined with certain therapy serving as control), and (5) depression evaluated preintervention and postintervention. We excluded studies that included patients with (1) a history of TMS therapy or (2) a metallic or electronic implant such as a pacemaker.

\section{Quality assessment}

Risk of bias of each included study was assessed using the RoB 2.0 tool. The domains evaluated were risk of bias in (1) the randomization process, (2) intended interventions, (3) missing data, (4) outcome measurement, and (5) selection of the reported result.

\section{Data extraction and analysis}

Reviewers extracted the following data: (1) patient characteristics, (2) number of patients receiving sham or rTMS treatment, (3) study design, (4) outcome measured, (5) rTMS protocol, and (6) standardized mean difference and standard error of change scores after interventions. Since current study used different scores or scales, we combined the effect size by the standardized mean difference, calculated from mean difference divided by standard deviation. Data extracted from the selected studies 
were analyzed using RevMan 5.3. We used the $\mathrm{I}^{2}$ test to examine heterogeneity. Data

were synthesized using a random-effects model.

\section{Reference}

1 Injury, G. B. D. T. B. \& Spinal Cord Injury, C. Global, regional, and national burden of traumatic brain injury and spinal cord injury, 1990-2016: a systematic analysis for the Global Burden of Disease Study 2016. Lancet Neurol 18, 56-87, doi:10.1016/S1474-4422(18)30415-0 (2019).

2 Dewan, M. C. et al. Estimating the global incidence of traumatic brain injury. J Neurosurg, 1-18, doi:10.3171/2017.10.JNS17352 (2018).

3 Bryant, R. A. et al. The psychiatric sequelae of traumatic injury. Am J Psychiatry 167, 312-320, doi:10.1176/appi.ajp.2009.09050617 (2010).

4 de Freitas Cardoso, M. G. et al. Cognitive Impairment Following Acute Mild Traumatic Brain Injury. Front Neurol 10, 198, doi:10.3389/fneur.2019.00198 (2019).

5 Ouellet, M. C. et al. Depression in the First Year after Traumatic Brain Injury. J Neurotrauma 35, 1620-1629, doi:10.1089/neu.2017.5379 (2018).

6 Jorge, R. E. et al. Major depression following traumatic brain injury. Arch Gen Psychiatry 61, 42-50, doi:10.1001/archpsyc.61.1.42 (2004).

7 Disease, G. B. D., Injury, I. \& Prevalence, C. Global, regional, and national incidence, prevalence, and years lived with disability for 354 diseases and injuries for 195 countries and territories, 1990-2017: a systematic analysis for the Global Burden of Disease Study 2017. Lancet 392, 1789-1858, doi:10.1016/S0140-6736(18)32279-7 (2018).

8 Rabinowitz, A. R. \& Levin, H. S. Cognitive sequelae of traumatic brain injury. Psychiatr Clin North Am 37, 1-11, doi:10.1016/j.psc.2013.11.004 (2014).

9 Novack, T. A., Alderson, A. L., Bush, B. A., Meythaler, J. M. \& Canupp, K. Cognitive and functional recovery at 6 and 12 months post-TBI. Brain Inj 14, 987-996, doi:10.1080/02699050050191922 (2000).

10 Skandsen, T. et al. Cognitive impairment 3 months after moderate and severe traumatic brain injury: a prospective follow-up study. Arch Phys Med Rehabil 91, 1904-1913, doi:10.1016/j.apmr.2010.08.021 (2010).

11 Grimm, S. et al. Imbalance between left and right dorsolateral prefrontal cortex in major depression is linked to negative emotional judgment: an fMRI 
study in severe major depressive disorder. Biol Psychiatry 63, 369-376, doi:10.1016/j.biopsych.2007.05.033 (2008).

12 Koenigs, M. \& Grafman, J. The functional neuroanatomy of depression: distinct roles for ventromedial and dorsolateral prefrontal cortex. Behav Brain Res 201, 239-243, doi:10.1016/j.bbr.2009.03.004 (2009).

13 Cole, M. W. \& Schneider, W. The cognitive control network: Integrated cortical regions with dissociable functions. Neuroimage 37, 343-360, doi:10.1016/j.neuroimage.2007.03.071 (2007).

$14 \mathrm{Li}, \mathrm{B}$. J. et al. A brain network model for depression: From symptom understanding to disease intervention. CNS Neurosci Ther 24, 1004-1019, doi:10.1111/cns.12998 (2018).

15 Wolf, J. A. \& Koch, P. F. Disruption of Network Synchrony and Cognitive Dysfunction After Traumatic Brain Injury. Front Syst Neurosci 10, 43, doi:10.3389/fnsys.2016.00043 (2016).

16 Siddiqi, S. H. et al. Repetitive Transcranial Magnetic Stimulation with RestingState Network Targeting for Treatment-Resistant Depression in Traumatic Brain Injury: A Randomized, Controlled, Double-Blinded Pilot Study. J Neurotrauma 36, 1361-1374, doi:10.1089/neu.2018.5889 (2019).

$17 \mathrm{Li}$, J. et al. Effects of different frequencies of repetitive transcranial magnetic stimulation on the recovery of upper limb motor dysfunction in patients with subacute cerebral infarction. Neural Regen Res 11, 1584-1590, doi:10.4103/1673-5374.193236 (2016).

18 Chou, Y. H., Hickey, P. T., Sundman, M., Song, A. W. \& Chen, N. K. Effects of repetitive transcranial magnetic stimulation on motor symptoms in Parkinson disease: a systematic review and meta-analysis. JAMA Neurol 72, 432-440, doi:10.1001/jamaneurol.2014.4380 (2015).

19 Liao, X. et al. Repetitive Transcranial Magnetic Stimulation as an Alternative Therapy for Cognitive Impairment in Alzheimer's Disease: A Meta-Analysis. J Alzheimers Dis 48, 463-472, doi:10.3233/JAD-150346 (2015).

20 Hsu, W. Y., Cheng, C. H., Liao, K. K., Lee, I. H. \& Lin, Y. Y. Effects of repetitive transcranial magnetic stimulation on motor functions in patients with stroke: a meta-analysis. Stroke 43, 1849-1857, doi:10.1161/STROKEAHA.111.649756 (2012).

21 Gaynes, B. N. et al. Repetitive transcranial magnetic stimulation for treatment-resistant depression: a systematic review and meta-analysis. J Clin Psychiatry 75, 477-489; quiz 489, doi:10.4088/JCP.13r08815 (2014).

22 Cao, X., Deng, C., Su, X. \& Guo, Y. Response and Remission Rates Following High-Frequency vs. Low-Frequency Repetitive Transcranial Magnetic 
Stimulation (rTMS) Over Right DLPFC for Treating Major Depressive Disorder (MDD): A Meta-Analysis of Randomized, Double-Blind Trials. Front Psychiatry 9, 413, doi:10.3389/fpsyt.2018.00413 (2018).

23 Moussavi, Z. et al. A Pilot Randomised Double-Blind Study of the Tolerability and efficacy of repetitive Transcranial Magnetic Stimulation on Persistent Post-Concussion Syndrome. Sci Rep 9, 5498, doi:10.1038/s41598-019-41923-6 (2019).

24 Hoy, K. E. et al. A Pilot Investigation of Repetitive Transcranial Magnetic Stimulation for Post-Traumatic Brain Injury Depression: Safety, Tolerability, and Efficacy. J Neurotrauma 36, 2092-2098, doi:10.1089/neu.2018.6097 (2019).

25 Lee, S. A. \& Kim, M. K. Effect of Low Frequency Repetitive Transcranial Magnetic Stimulation on Depression and Cognition of Patients with Traumatic Brain Injury: A Randomized Controlled Trial. Med Sci Monit 24, 8789-8794, doi:10.12659/MSM.911385 (2018).

26 Rao, V. et al. Low-Frequency Right Repetitive Transcranial Magnetic Stimulation for the Treatment of Depression After Traumatic Brain Injury: A Randomized Sham-Controlled Pilot Study. J Neuropsychiatry Clin Neurosci 31, 306-318, doi:10.1176/appi.neuropsych.17110338 (2019).

27 Leung, A. et al. Left Dorsolateral Prefrontal Cortex rTMS in Alleviating MTBI Related Headaches and Depressive Symptoms. Neuromodulation 21, 390-401, doi:10.1111/ner.12615 (2018).

28 Stilling, J. et al. Treatment of Persistent Post-Traumatic Headache and PostConcussion Symptoms Using Repetitive Transcranial Magnetic Stimulation: A Pilot, Double-Blind, Randomized Controlled Trial. J Neurotrauma 37, 312-323, doi:10.1089/neu.2019.6692 (2020).

29 Perianez, J. A. et al. Trail Making Test in traumatic brain injury, schizophrenia, and normal ageing: sample comparisons and normative data. Arch Clin Neuropsychol 22, 433-447, doi:10.1016/j.acn.2007.01.022 (2007).

30 Scarpina, F. \& Tagini, S. The Stroop Color and Word Test. Front Psychol 8, 557, doi:10.3389/fpsyg.2017.00557 (2017).

31 Tam, J. W. \& Schmitter-Edgecombe, M. The role of processing speed in the Brief Visuospatial Memory Test - revised. Clin Neuropsychol 27, 962-972, doi:10.1080/13854046.2013.797500 (2013).

32 King, N. S., Crawford, S., Wenden, F. J., Moss, N. E. \& Wade, D. T. The Rivermead Post Concussion Symptoms Questionnaire: a measure of symptoms commonly experienced after head injury and its reliability. J Neurol 242, 587-592, doi:10.1007/BF00868811 (1995). 
33 Zhang, Y. Q. et al. Bilateral repetitive transcranial magnetic stimulation for treatment-resistant depression: a systematic review and meta-analysis of randomized controlled trials. Braz J Med Biol Res 48, 198-206, doi:10.1590/1414-431X20144270 (2015).

34 Trevizol, A. P. et al. Unilateral and bilateral repetitive transcranial magnetic stimulation for treatment-resistant late-life depression. Int J Geriatr Psychiatry 34, 822-827, doi:10.1002/gps.5091 (2019).

35 Martin, D. M., McClintock, S. M., Forster, J. J., Lo, T. Y. \& Loo, C. K. Cognitive enhancing effects of rTMS administered to the prefrontal cortex in patients with depression: A systematic review and meta-analysis of individual task effects. Depress Anxiety 34, 1029-1039, doi:10.1002/da.22658 (2017).

36 Koski, L. et al. Noninvasive brain stimulation for persistent postconcussion symptoms in mild traumatic brain injury. J Neurotrauma 32, 38-44, doi:10.1089/neu.2014.3449 (2015).

37 Guse, B., Falkai, P. \& Wobrock, T. Cognitive effects of high-frequency repetitive transcranial magnetic stimulation: a systematic review. J Neural Transm (Vienna) 117, 105-122, doi:10.1007/s00702-009-0333-7 (2010).

38 Kim, T. D., Hong, G., Kim, J. \& Yoon, S. Cognitive Enhancement in Neurological and Psychiatric Disorders Using Transcranial Magnetic Stimulation (TMS): A Review of Modalities, Potential Mechanisms and Future Implications. Exp Neurobiol 28, 1-16, doi:10.5607/en.2019.28.1.1 (2019).

39 Guan, H. Y. et al. High-frequency neuronavigated rTMS effect on clinical symptoms and cognitive dysfunction: a pilot double-blind, randomized controlled study in Veterans with schizophrenia. Transl Psychiatry 10, 79, doi:10.1038/s41398-020-0745-6 (2020).

40 Teng, S. et al. High-frequency repetitive transcranial magnetic stimulation over the left DLPFC for major depression: Session-dependent efficacy: A meta-analysis. Eur Psychiatry 41, 75-84, doi:10.1016/j.eurpsy.2016.11.002 (2017).

41 Schulze, L. et al. Number of pulses or number of sessions? An open-label study of trajectories of improvement for once-vs. twice-daily dorsomedial prefrontal rTMS in major depression. Brain Stimul 11, 327-336, doi:10.1016/j.brs.2017.11.002 (2018).

42 Pink, A. E., Williams, C., Alderman, N. \& Stoffels, M. The use of repetitive transcranial magnetic stimulation (rTMS) following traumatic brain injury (TBI): A scoping review. Neuropsychol Rehabil, 1-27, doi:10.1080/09602011.2019.1706585 (2019). 


\section{Acknowledgements}

1. This work was partially supported by the Ministry of Science and Technology of Taiwan (grant number MOST-109-2221-E-038-006).

2. This manuscript was edited by Wallace Academic Editing.

\section{Author contributions}

PYT performed the data collection, data analysis and manuscript draft. YCC

contributed to the statistical analysis, data collection and quality assessment. JYW executed the data selection, quality assessment as well as manuscript revision. KHC interpreted the data and drafted the manuscript. CHL contributed substantially to the study design, quality assessment, manuscript draft, and manuscript revision. All authors contributed to the writing of the manuscript and approved the final version.

\section{Additional information}

The Authors declare that there is no conflict of interest. 


\begin{tabular}{|c|c|c|c|c|c|c|c|c|c|}
\hline \multirow{2}{*}{$\begin{array}{l}\text { Author } \\
\text { (year) }\end{array}$} & \multirow[t]{2}{*}{ Patient population } & \multirow{2}{*}{$\begin{array}{l}\text { Mean } \\
\text { age }\end{array}$} & \multirow[t]{2}{*}{ Men } & \multirow[t]{2}{*}{ Women } & \multirow{2}{*}{$\begin{array}{l}\text { Number of participants in } \\
\text { the control and } \\
\text { intervention groups }\end{array}$} & \multirow[t]{2}{*}{ Study design } & \multicolumn{3}{|c|}{ Outcomes measured and parameters used } \\
\hline & & & & & & & \begin{tabular}{|l|} 
Depression \\
average score \\
(baseline) \\
\end{tabular} & Cognition & $\begin{array}{l}\text { Postconcussion } \\
\text { syndrome }\end{array}$ \\
\hline $\begin{array}{l}\text { Stilling } \\
(2020)\end{array}$ & $\begin{array}{l}\text { Mild TBI with PTH } \\
\text { and PPCS }\end{array}$ & 36 & 2 & 18 & $\begin{array}{l}\text { Sham rTMS N }=10 \\
\text { Real rTMS N }=10\end{array}$ & $\begin{array}{l}\text { Double- } \\
\text { blinded RCT }\end{array}$ & $\begin{array}{l}\text { PHQ-9 } \\
11.9\end{array}$ & - & RPQ-3, RPQ-13 \\
\hline $\begin{array}{l}\text { Moussavi } \\
(2019)\end{array}$ & *TBI with PPCS & 45.9 & 2 & 6 & $\begin{array}{l}\text { Sham rTMS N }=4 \\
\text { Real rTMS N }=4\end{array}$ & $\begin{array}{l}\text { Double- } \\
\text { blinded RCT }\end{array}$ & $\begin{array}{l}\text { MADRS } \\
19.65 \\
\end{array}$ & - & RPQ-3, RPQ-13 \\
\hline $\begin{array}{l}\text { Rao } \\
(2019)\end{array}$ & TBI with depression & 40.0 & 16 & 14 & $\begin{array}{l}\text { Sham rTMS N }=17 \\
\text { Real rTMS N }=13\end{array}$ & $\begin{array}{l}\text { Double- } \\
\text { blinded RCT }\end{array}$ & $\begin{array}{l}\text { HAMD } \\
23.5 \\
\end{array}$ & $\begin{array}{l}\text { TMT-A, TMT-B, } \\
\text { BVMT, SCWT } \\
\end{array}$ & RPQ-13 \\
\hline $\begin{array}{l}\text { Hoy } \\
(2019)\end{array}$ & $\begin{array}{l}\text { TBI with moderate to } \\
\text { severe depression }\end{array}$ & 46.3 & 10 & 11 & $\begin{array}{l}\text { Sham rTMS N }=10 \\
\text { Real rTMS N }=11\end{array}$ & $\begin{array}{l}\text { Double- } \\
\text { blinded RCT }\end{array}$ & $\begin{array}{l}\text { MADRS } \\
34.0 \\
\end{array}$ & $\begin{array}{l}\text { TMT-A, TMT-B, } \\
\text { BVMT, SCWT }\end{array}$ & - \\
\hline $\begin{array}{l}\text { Siddiqi } \\
\text { (2019) }\end{array}$ & $\begin{array}{l}\text { Moderate TBI with } \\
\text { treatment-resistant } \\
\text { depression }\end{array}$ & 45.8 & 11 & 4 & $\begin{array}{l}\text { Sham rTMS N }=6 \\
\text { Real rTMS N }=9\end{array}$ & $\begin{array}{l}\text { Double- } \\
\text { blinded RCT }\end{array}$ & $\begin{array}{l}\text { MADRS } \\
32.2\end{array}$ & - & - \\
\hline $\begin{array}{l}\text { Lee } \\
(2018)\end{array}$ & $\begin{array}{l}\text { TBI with mild to } \\
\text { moderate depression }\end{array}$ & 41.9 & 9 & 4 & $\begin{array}{l}\text { NDT + Sham rTMS N }=6 \\
\text { NDT + Real rTMS N }=7\end{array}$ & $\begin{array}{l}\text { Single- } \\
\text { blinded RCT }\end{array}$ & $\begin{array}{l}\text { MADRS } \\
23.8 \\
\end{array}$ & TMT, SCWT & - \\
\hline $\begin{array}{l}\text { Leung } \\
(2018)\end{array}$ & Mild TBI with PTH & 34.1 & 23 & 6 & $\begin{array}{l}\text { Sham rTMS N }=15 \\
\text { Real rTMS N = } 14\end{array}$ & $\begin{array}{l}\text { Double- } \\
\text { blinded RCT }\end{array}$ & \begin{tabular}{|l} 
HAMD \\
23.9
\end{tabular} & - & - \\
\hline
\end{tabular}

\section{Table 1. Summary of study characteristics and outcomes measured}

*Only people having severe postconcussion syndrome (SPCS) were included according to inclusion criteria.

Abbreviation: $\mathrm{TBI}=$ traumatic brain injury, $\mathrm{PTH}=$ posttraumatic headache, $\mathrm{PPCS}=$ persistent postconcussion syndrome, NDT $=$ neurodevelopmental therapy, rTMS = repetitive transcranial magnetic stimulation, RPQ = Rivermead Post-Concussion Symptom Questionnaire, MADRS = Montgomery-Asberg Depression Rating Scale, HAMD = Hamilton Rating Scale for Depression, PHQ-9 = Patient Health Questionnaire 9, TMT = Trail Making Test, BVMT = Brief Visuospatial Memory Test, $\mathrm{SCWT}=$ Stroop Color Word Test, $\mathrm{RCT}=$ randomized controlled trial 


\begin{tabular}{|l|l|l|l|l|l|l|l|l|}
\hline Author/year & Coil type & Location & $\begin{array}{l}\text { Frequency } \\
(\mathrm{Hz})\end{array}$ & $\begin{array}{l}\text { Intensity }(\% \\
\mathrm{RMT})\end{array}$ & Sessions & $\begin{array}{l}\text { Pulses } \times \\
\text { train(s)/session }\end{array}$ & $\begin{array}{l}\text { Duration of } \\
\text { each train }(\mathrm{s})\end{array}$ & $\begin{array}{l}\text { Intertrain } \\
\text { interval }(\mathrm{s})\end{array}$ \\
\hline $\begin{array}{l}\text { Stilling } \\
(2020)\end{array}$ & $\begin{array}{l}\text { Figure of } 8 \\
\text { coil }\end{array}$ & Left DLPFC & 10 & 70 & 10 & $60 \times 10$ & 6 \\
\hline $\begin{array}{l}\text { Moussavi } \\
(2019)\end{array}$ & $\begin{array}{l}\text { Figure of } 8 \\
\text { coil }\end{array}$ & Left DLPFC & 20 & 100 & 13 & $30 \times 25$ & 1.5 \\
\hline $\begin{array}{l}\text { Rao } \\
(2019)\end{array}$ & $\begin{array}{l}\text { Figure of } 8 \\
\text { coil }\end{array}$ & Right DLPFC & 1 & 110 & 20 & $300 \times 4$ & 300 \\
\hline $\begin{array}{l}\text { Hoy } \\
(2019)\end{array}$ & $\begin{array}{l}\text { Figure of } 8 \\
\text { coil }\end{array}$ & $\begin{array}{l}\text { Bilateral sequential } \\
\text { DLPFC (right } \rightarrow \text { left) }\end{array}$ & $\begin{array}{l}\text { Right: } 1 \\
\text { Left: } 10\end{array}$ & 110 & 20 & $\begin{array}{l}\text { Right: } 900 \times 1 \\
\text { Left: } 50 \times 30\end{array}$ & 5 \\
\hline $\begin{array}{l}\text { Siddiqi } \\
(2019)\end{array}$ & NA & $\begin{array}{l}\text { Bilateral sequential } \\
\text { DLPFC }(\text { left } \rightarrow \text { right })\end{array}$ & $\begin{array}{l}\text { Right: } 1 \\
\text { Left: } 10\end{array}$ & 120 & 20 & $\begin{array}{l}\text { Right: } 1000 \times 1 \\
\text { Left: } 50 \times 80\end{array}$ & 5 \\
\hline $\begin{array}{l}\text { Lee } \\
(2018)\end{array}$ & $\begin{array}{l}\text { Figure of } 8 \\
\text { coil }\end{array}$ & Right DLPFC & 1 & 100 & 10 & $40 \times 50$ & $\begin{array}{l}\text { Right: nil } \\
\text { Left: } 25\end{array}$ \\
\hline $\begin{array}{l}\text { Leung } \\
(2018)\end{array}$ & $\begin{array}{l}\text { Figure of } 8 \\
\text { coil }\end{array}$ & Left DLPFC & 10 & 80 & 4 & 40 & 25 \\
\hline
\end{tabular}

\section{Table 2. Summary of rTMS protocols in included studies}

Abbreviation: DLPFC $=$ dorsolateral prefrontal cortex, $\mathrm{RMT}=$ resting motor threshold 
(a)

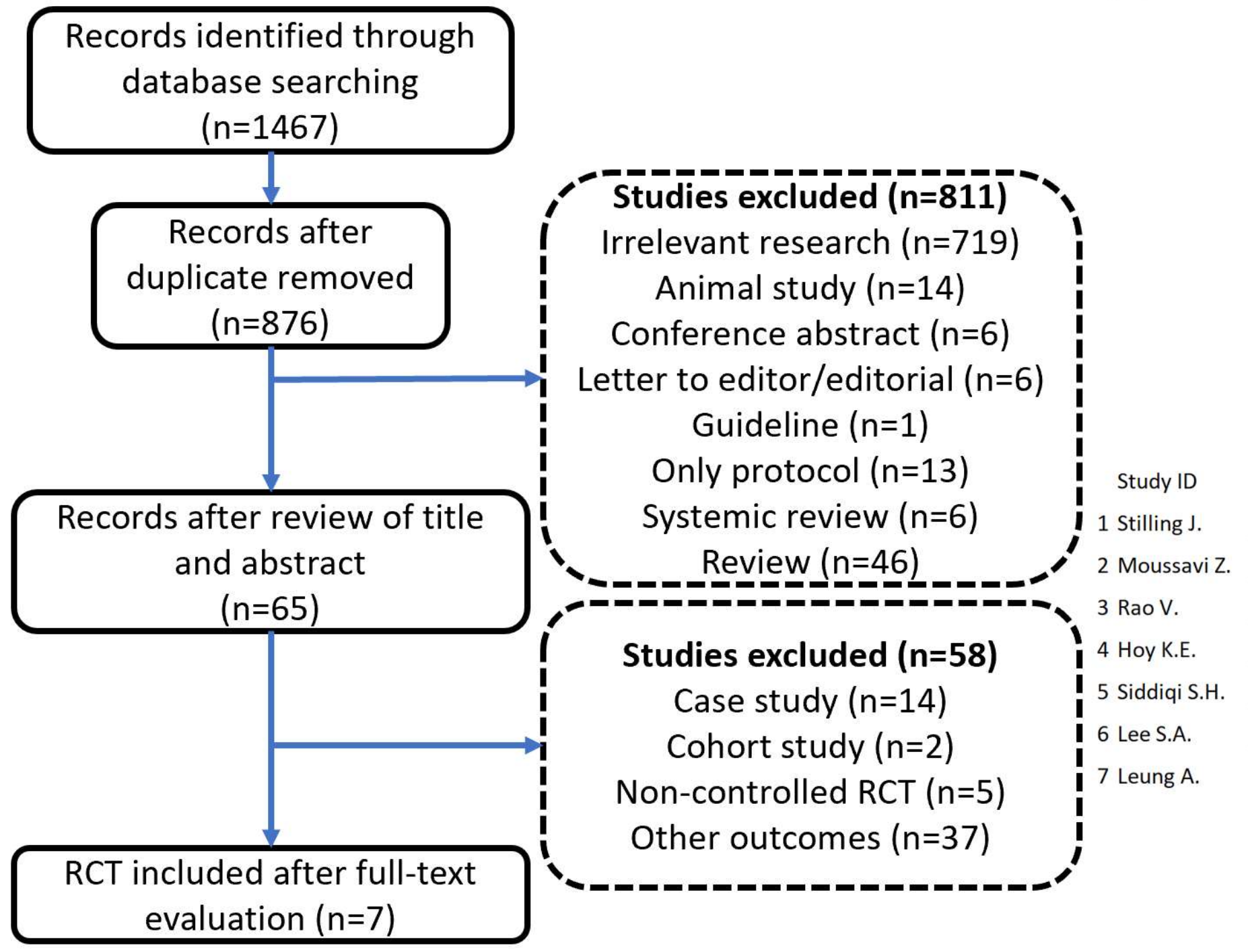

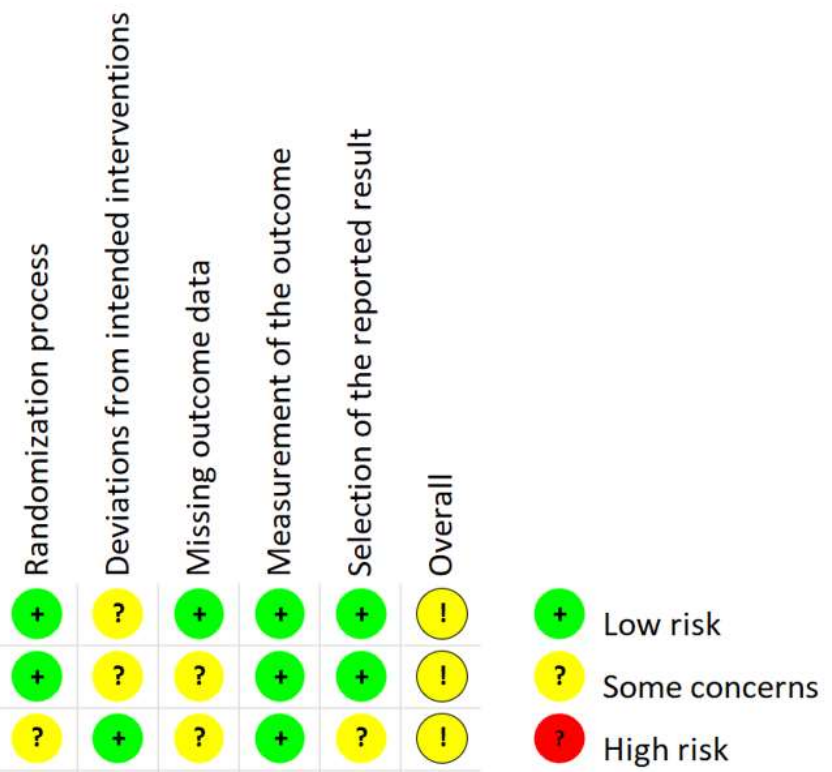

Figure 1. Flowchart of study selection and risk of bias of included studies

(a) Flowchart and (b) risk of bias 
(a) Immediately after rTMS treatment

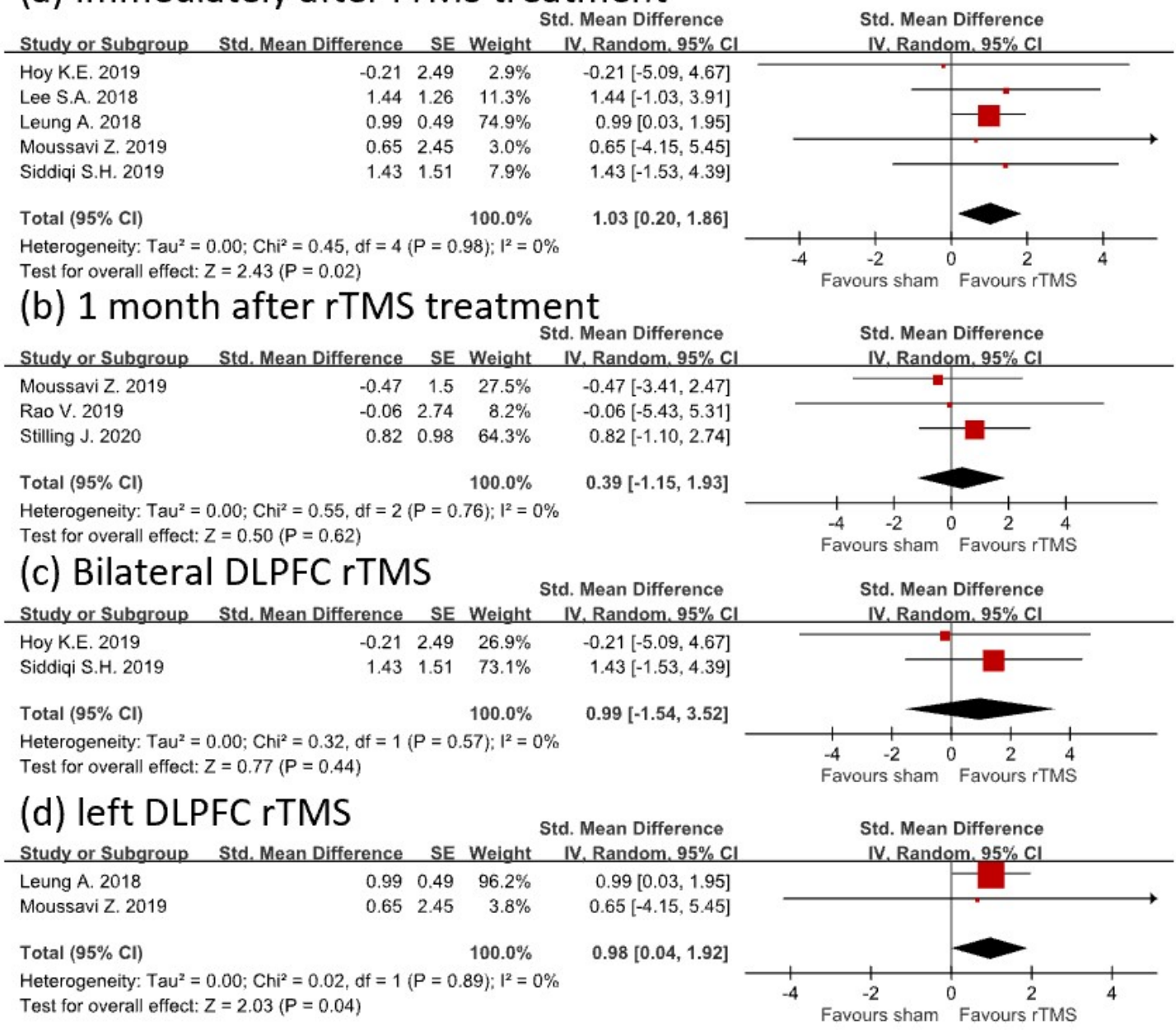

\section{(e) Right DLPFC rTMS}

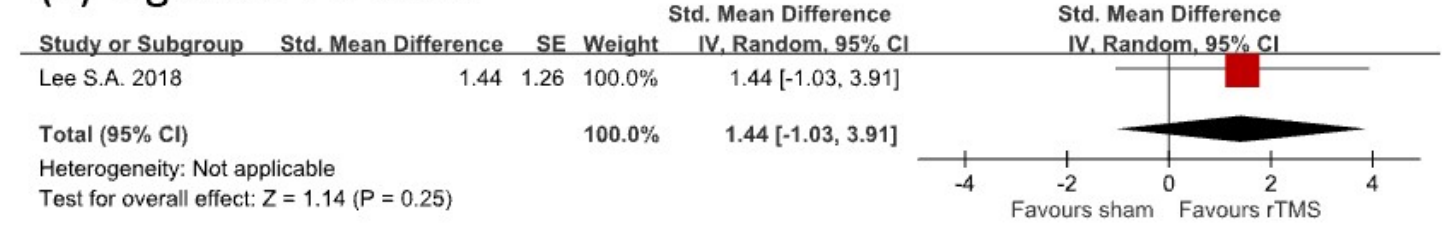

Figure 2. Forest plot showing the efficacy of rTMS versus sham in treating depression

The depression outcome was assessed (a) immediately and (b) 1 month after rTMS treatment. In the subgroup analysis, the depression outcome was measured immediately after (c) bilateral, (d) left, and (e) right rTMS treatment. 
(a) TMT-A

Std. Mean Difference

Study or Subgroup Std. Mean Difference SE Weight IV. Random. 95\%

Hoy K.E. 2019

$\begin{array}{lll}-0.14 & 4.21 \quad 0.2 \%\end{array}$

Rao V. 2019

$-0.25 \quad 0.19 \quad 99.8 \%$

$-0.14[-8.39,8.11]$

$-0.25[-0.62,0.12]$

Total $(95 \% \mathrm{Cl})$

$100.0 \%$

Heterogeneity: $\mathrm{Tau}^{2}=0.00 ; \mathrm{Chi}^{2}=0.00, \mathrm{df}=1(\mathrm{P}=0.98) ; \mathrm{I}^{2}=0 \%$

Test for overall effect: $Z=1.32(P=0.19)$

(b) TMT-B

$-0.25[-0.62,0.12]$

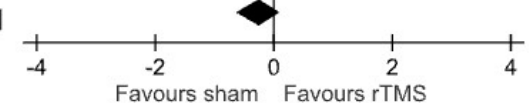

Study or Subgroup Std. Mean Difference SE Weight

$\begin{array}{lllll}\text { Hoy K.E. } 2019 & 0.22 & 3.8 & 3.7 \% & 0.22[-7.23,7.67]\end{array}$

$\begin{array}{lllll}\text { Rao V. } 2019 & -0.28 & 0.75 & 96.3 \% & -0.28[-1.75,1.19]\end{array}$

Total $(95 \% \mathrm{Cl}) \quad 100.0 \%$

Heterogeneity: $\mathrm{Tau}^{2}=0.00 ; \mathrm{Chi}^{2}=0.02, \mathrm{df}=1(\mathrm{P}=0.90) ; \mathrm{I}^{2}=0 \%$

Test for overall effect: $Z=0.36(P=0.72)$

(c) TMT-A+B

$-0.26[-1.70,1.18]$

Study or Subgroup

Std. Mean Difference SE Weigh

Std. Mean Difference

Std. Mean Difference

Hoy K.E. 2019 (TMT-A)

Hoy K.E. 2019 (TMT-B)

Lee S.A. 2018

Rao V. 2019 (TMT-A)

Rao V. 2019 (TMT-B)

$0.22 \quad 3.8 \quad 0.2 \%$

$\begin{array}{lll}1.49 & 0.9 & 4.0 \%\end{array}$

$\begin{array}{lll}-0.25 & 0.19 \quad 89.8 \%\end{array}$

$\begin{array}{rrr}-0.25 & 0.19 & 89.8 \% \\ -0.28 & 0.75 & 5.8 \%\end{array}$

Total $(95 \% \mathrm{Cl})$

$100.0 \%$

Heterogeneity: $\mathrm{Tau}^{2}=0.00 ; \mathrm{Chi}^{2}=3.61, \mathrm{df}=4(\mathrm{P}=0.46) ; \mathrm{I}^{2}=0 \%$ Test for overall effect: $Z=1.00(P=0.32)$

(d) SCWT

IV. Random. $95 \%$ C

$-0.14[-8.39,8.11]$

$0.22[-7.23,7.67]$

$1.49[-0.27,3.25]$

$-0.25[-0.62,0.12]$

$-0.28[-1.75,1.19]$

$-0.18[-0.53,0.17]$

IV. Random. $95 \% \mathrm{C}$
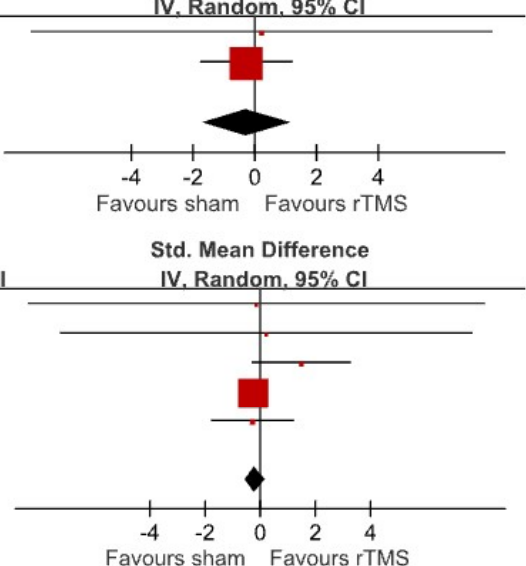

Favours sham Favours rTMS

Std. Mean Difference SE Weight

2019

Lee S.A. 2018

Std. Mean Difference

Std. Mean Difference

Rao V. 2019

$\begin{array}{rrr}0.03 & 12.4 & 0.0 \% \\ 1.24 & 3.8 & 0.3 \%\end{array}$

$0.21 \quad 0.21 \quad 99.7 \%$

Random. 95\%

$0.03[-24.27,24.33]$

$1.24[-6.21,8.69]$

$0.21[-0.20,0.62]$

Total $(95 \% \mathrm{Cl})$

$100.0 \%$

Heterogeneity: $\mathrm{Tau}^{2}=0.00 ; \mathrm{Chi}^{2}=0.07, \mathrm{df}=2(\mathrm{P}=0.96) ; \mathrm{I}^{2}=0 \%$

Test for overall effect: $Z=1.02(P=0.31)$

(e) BVMT

$0.21[-0.20,0.62]$

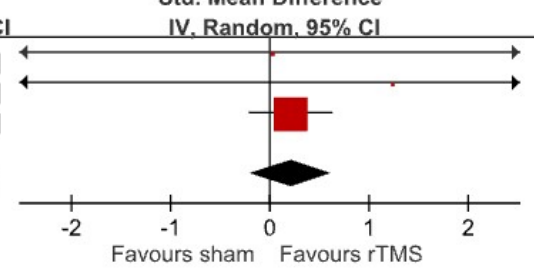

Std. Mean Difference
Study or Subgroun Std. Mean Difference SE Weight IV Random $95 \%$

Hoy K.E. 2019

$\begin{array}{lll}0.39 & 0.09 & 99.8 \%\end{array}$

Rao V. 2019

$-0.33 \quad 2.05$

$0.2 \%$

Total $(95 \% \mathrm{Cl})$

$100.0 \%$

Heterogeneity: $\mathrm{Tau}^{2}=0.00 ; \mathrm{Chi}^{2}=0.12, \mathrm{df}=1(\mathrm{P}=0.73) ; \mathrm{I}^{2}=0 \%$

Test for overall effect: $Z=4.32(P<0.0001)$

$0.39[0.21,0.57]$

$-0.33[-4.35,3.69]$

$0.39[0.21,0.56]$

Std. Mean Difference

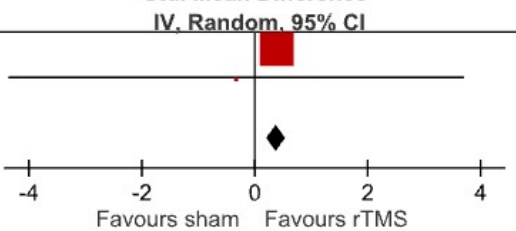

Figure 3. Forest plot showing efficacy of treatment versus sham treatment in improving cognitive function

Different cognitive domains, including $(\mathrm{a}-\mathrm{c})$ processing speed, (d) selective attention, and (e) visuospatial memory, were evaluated. 
(a) RPQ-3

Std. Mean Difference

Study or Subgroup Std. Mean Difference SE Weight

Moussavi Z. 2019

Stilling J. 2020

$\begin{array}{lll}-0.1 & 0.75 & 21.3 \%\end{array}$

$\begin{array}{lll}0.46 & 0.39 & 78.7 \%\end{array}$

V. Random. $95 \% \mathrm{Cl}$

IV. Random. $95 \%$ CI

Total $(95 \% \mathrm{Cl})$

$100.0 \%$

Heterogeneity: $\mathrm{Tau}^{2}=0.00 ; \mathrm{Chi}^{2}=0.44, \mathrm{df}=1(\mathrm{P}=0.51) ; \mathrm{I}^{2}=0 \%$

Test for overall effect: $Z=0.98(P=0.32)$

(b) RPQ-13

$-0.10[-1.57,1.37]$

$0.46[-0.30,1.22]$

Study or Subgroup

$0.34[-0.34,1.02]$

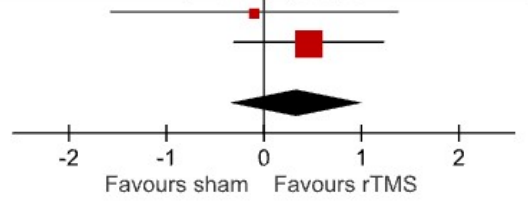

Moussavi Z. 2019

Rao V. 2019

Std. Mean Difference

Std. Mean Difference

Stilling J. 2020 $\begin{array}{lll}0.7 & 2.38 & 1.0 \%\end{array}$ $\begin{array}{lll}-0.53 & 0.24 \quad 97.6 \%\end{array}$ $0.48 \quad 2 \quad 1.4 \%$

IV. Random. $95 \% \mathrm{Cl}$ IV. Random, $95 \% \mathrm{Cl}$

Total $(95 \% \mathrm{Cl}) \quad 100.0 \%$

Heterogeneity: $\mathrm{Tau}^{2}=0.00 ; \mathrm{Chi}^{2}=0.51, \mathrm{df}=2(\mathrm{P}=0.78) ; \mathrm{I}^{2}=0 \%$

Test for overall effect: $Z=2.12(P=0.03)$

$0.70[-3.96,5.36]$

$-0.53[-1.00,-0.06]$

$0.48[-3.44,4.40]$

$-0.50[-0.97,-0.04]$

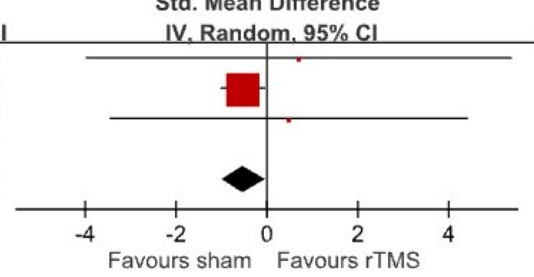

Figure 4. Forest plot showing efficacy of treatment versus sham treatment in postconcussion syndrome

(a) RPQ-3 and (b) RPQ-13 
Figures

(a)

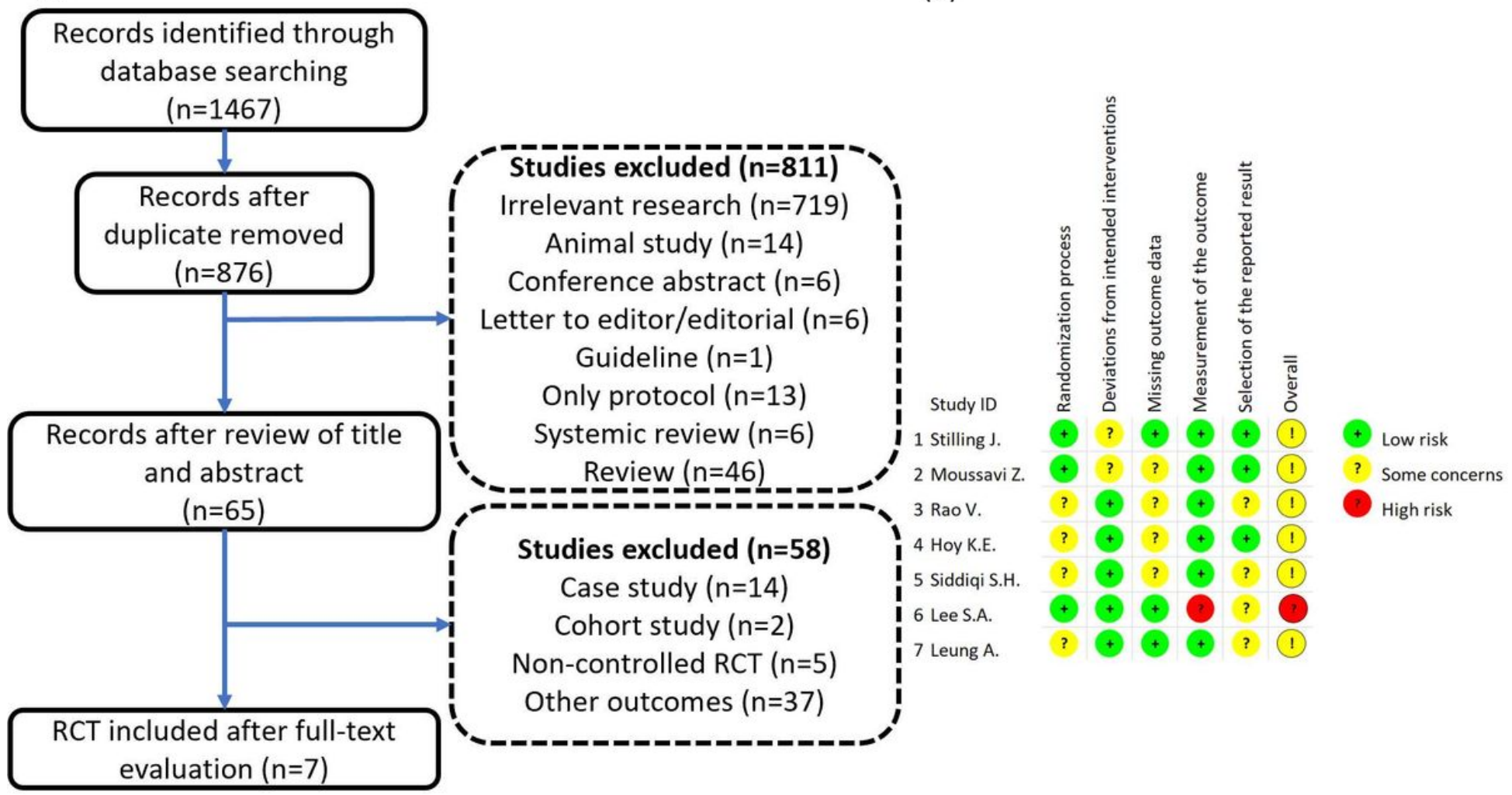

\section{Figure 1}

Flowchart of study selection and risk of bias of included studies (a) Flowchart and (b) risk of bias 


\section{(a) Immediately after rTMS treatment}

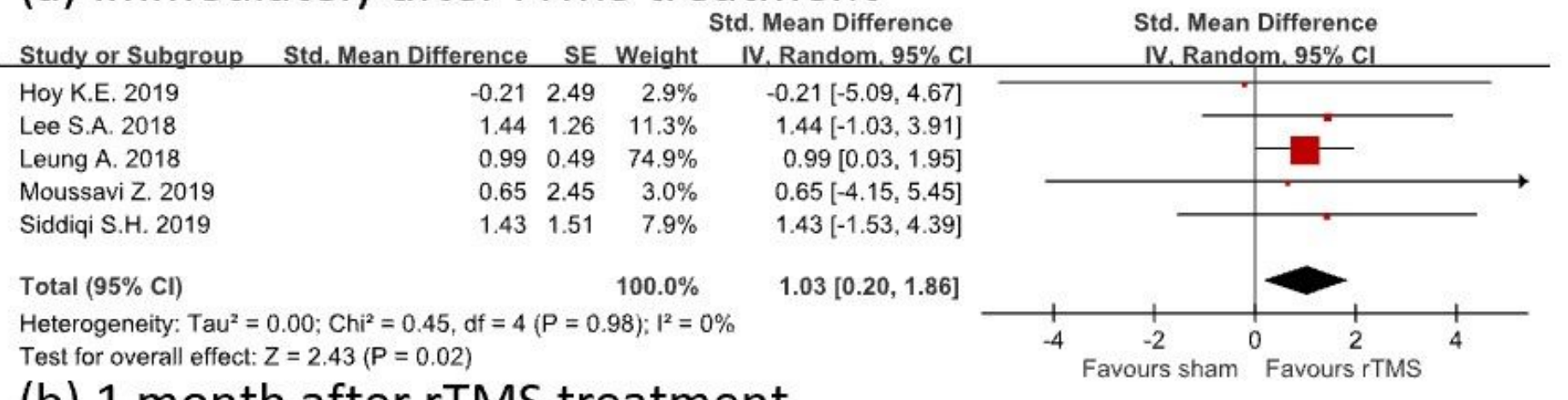

\section{(b) 1 month after rTMS treatment}

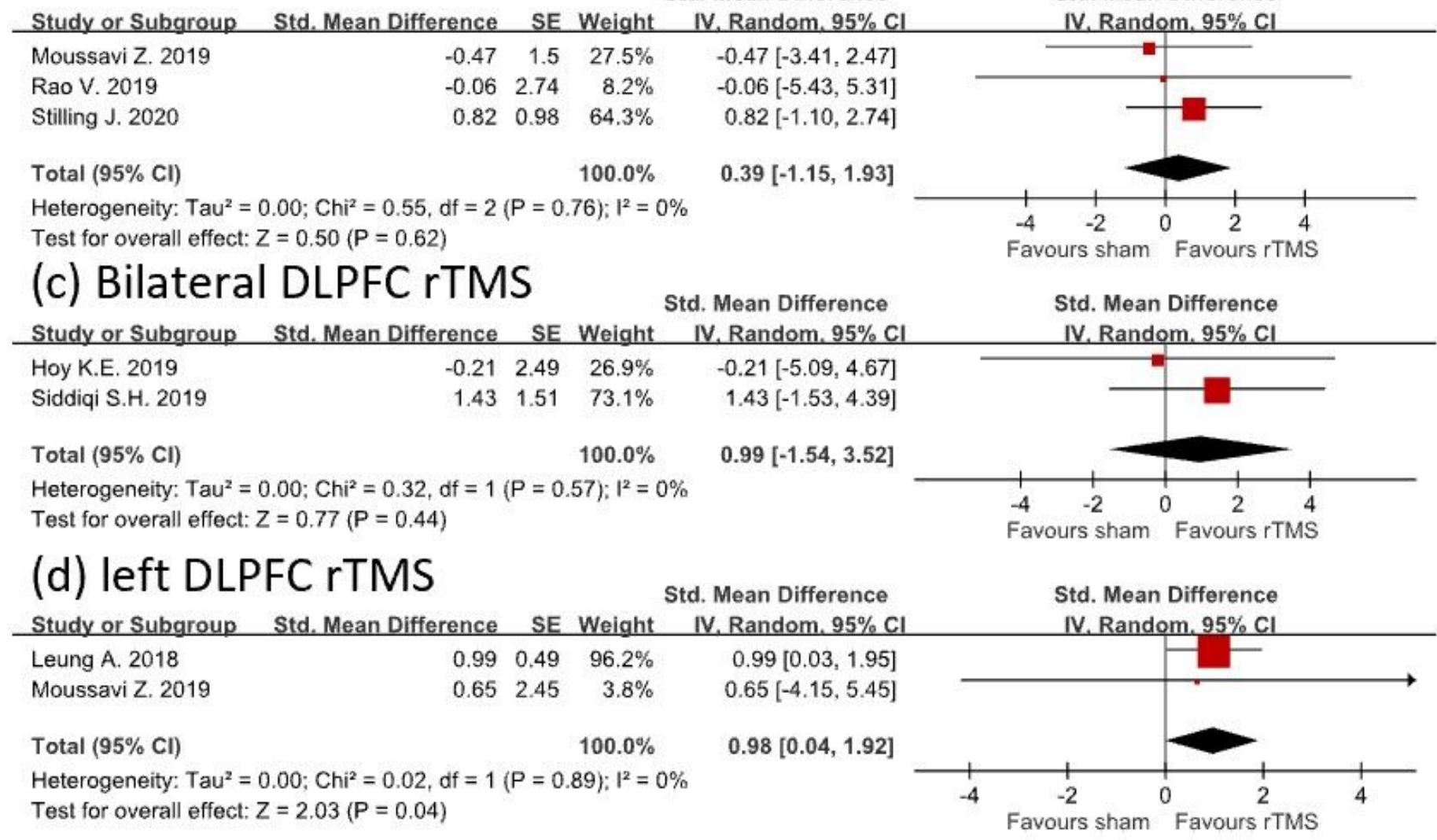

\section{(e) Right DLPFC rTMS}

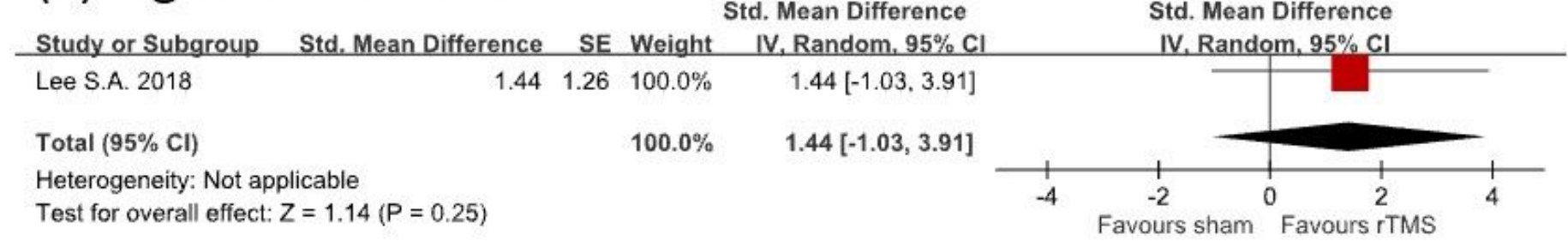

\section{Figure 2}

Forest plot showing the efficacy of rTMS versus sham in treating depression The depression outcome was assessed (a) immediately and (b) 1 month after rTMS treatment. In the subgroup analysis, the depression outcome was measured immediately after (c) bilateral, (d) left, and (e) right rTMS treatment. 


\section{(a) TMT-A}

Std. Mean Difference

Std. Mean Difference

Study or Subgroup

Std. Mean Difference SE Weight

IV. Random. $95 \% \mathrm{Cl}$

IV. Random. $95 \% \mathrm{Cl}$

Hoy K.E. 2019

$\begin{array}{lll}-0.14 & 4.21 \quad 0.2 \%\end{array}$

Rao V. 2019

$\begin{array}{lll}-0.25 & 0.19 \quad 99.8 \%\end{array}$

$-0.14[-8.39,8.11]$

$-0.25[-0.62,0.12]$

Total $(95 \% \mathrm{CI})$

$100.0 \%$

$-0.25[-0.62,0.12]$

Heterogeneity: $\mathrm{Tau}^{2}=0.00 ; \mathrm{Chi}^{2}=0.00, \mathrm{df}=1(\mathrm{P}=0.98) ; \mathrm{I}^{2}=0 \%$

Test for overall effect: $Z=1.32(P=0.19)$

\section{(b) TMT-B}

Std. Mean Difference

Study or Subgroup Std. Mean Difference SE Weight IV. Random. $95 \% \mathrm{CI}$

Hoy K.E. 2019

Rao V. 2019

$\begin{array}{lll}0.22 & 3.8 & 3.7 \%\end{array}$

$0.22[-7.23,7.67]$

$\begin{array}{lll}-0.28 & 0.75 & 96.3 \%\end{array}$

$-0.28[-1.75,1.19]$

Total $(95 \% \mathrm{Cl})$

$100.0 \%$

Heterogeneity: $\mathrm{Tau}^{2}=0.00 ; \mathrm{Chi}^{2}=0.02, \mathrm{df}=1(\mathrm{P}=0.90) ; \mathrm{I}^{2}=0 \%$

$-0.26[-1.70,1.18]$

Test for overall effect: $Z=0.36(P=0.72)$

\section{(c) TMT-A+B}

Std. Mean Difference

Study or Subgroup

Std. Mean Difference SE Weight

IV. Random, $95 \% \mathrm{Cl}$

\section{Hoy K.E. 2019 (TMT-A)}

Hoy K.E. 2019 (TMT-B)

Lee S.A. 2018

$-0.14 \quad 4.21 \quad 0.2 \%$

$0.22 \quad 3.8 \quad 0.2 \%$

$-0.14[-8.39,8.11]$

$0.22[-7.23,7.67]$

$\begin{array}{lll}1.49 & 0.9 & 4.0 \%\end{array}$

Rao V. 2019 (TMT-A)

Rao V. 2019 (TMT-B)

$\begin{array}{lll}-0.25 & 0.19 \quad 89.8 \%\end{array}$

$\begin{array}{lll}-0.28 & 0.75 \quad 5.8 \%\end{array}$

$1.49[-0.27,3.25]$

$-0.25[-0.62,0.12]$

$-0.28[-1.75,1.19]$

Total $(95 \% \mathrm{Cl})$

$100.0 \%$

Heterogeneity: $\mathrm{Tau}^{2}=0.00 ; \mathrm{Chi}^{2}=3.61, \mathrm{df}=4(\mathrm{P}=0.46) ; \mathrm{I}^{2}=0 \%$

Test for overall effect: $Z=1.00(P=0.32)$

(d) SCWT

$-0.18[-0.53,0.17]$

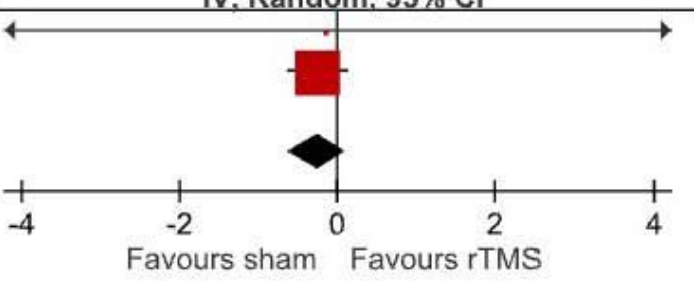

Favours sham Favours rTMS

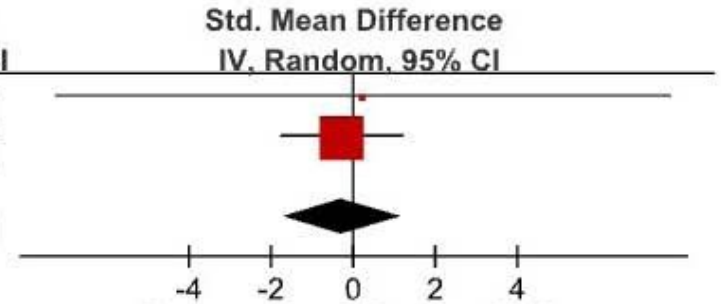

Study or Subgroup Std. Mean Difference SE Weight

Std. Mean Difference

Std. Mean Difference

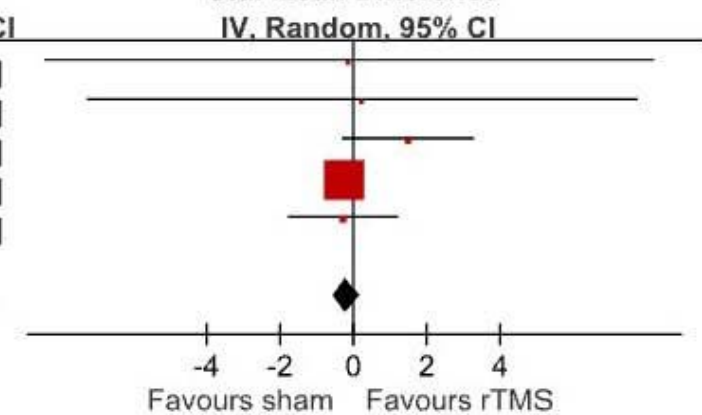

Hoy K.E. 2019

Lee S.A. 2018

$\begin{array}{lll}0.03 & 12.4 & 0.0 \%\end{array}$

IV. Random. $95 \% \mathrm{Cl}$

Rao V. 2019

$\begin{array}{lll}1.24 & 3.8 & 0.3 \%\end{array}$

$0.03[-24.27,24.33]$

$1.24[-6.21,8.69]$

$0.21[-0.20,0.62]$

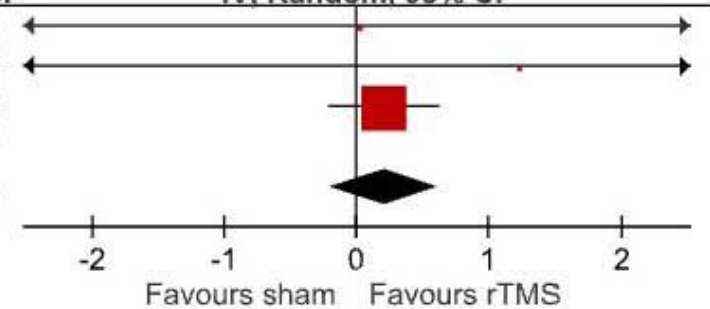

Total $(95 \% \mathrm{CI})$

$100.0 \%$

Heterogeneity: $\mathrm{Tau}^{2}=0.00 ; \mathrm{Chi}^{2}=0.07, \mathrm{df}=2(\mathrm{P}=0.96) ; \mathrm{I}^{2}=0 \%$

Test for overall effect: $Z=1.02(P=0.31)$

$0.21[-0.20,0.62]$

(e) BVMT

Std. Mean Difference

Study or Subgroup Std. Mean Difference SE Weight

IV. Random. $95 \% \mathrm{Cl}$

Hoy K.E. 2019

$\begin{array}{lll}0.39 & 0.09 \quad 99.8 \%\end{array}$

Rao V. 2019

$-0.33 \quad 2.05$

$0.2 \%$

$0.39[0.21,0.57]$

$-0.33[-4.35,3.69]$

Total $(95 \% \mathrm{Cl})$ $100.0 \%$

Heterogeneity: $\mathrm{Tau}^{2}=0.00 ; \mathrm{Chi}^{2}=0.12, \mathrm{df}=1(\mathrm{P}=0.73) ; \mathrm{I}^{2}=0 \%$

Test for overall effect: $Z=4.32(P<0.0001)$

$0.39[0.21,0.56]$

Std. Mean Difference IV. Random. $95 \% \mathrm{Cl}$

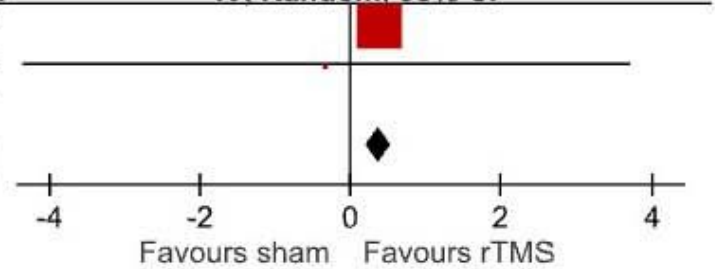

\section{Figure 3}

Forest plot showing efficacy of treatment versus sham treatment in improving cognitive function Different cognitive domains, including $(a-c)$ processing speed, (d) selective attention, and (e) visuospatial memory, were evaluated. 


\section{(a) RPQ-3}

Study or Subgroup Std. Mean Difference SE Weight

Std. Mean Difference

IV. Random. $95 \% \mathrm{Cl}$

$\begin{array}{lll}-0.1 & 0.75 & 21.3 \%\end{array}$

$\begin{array}{lll}0.46 & 0.39 & 78.7 \%\end{array}$

$100.0 \%$

Total $(95 \% \mathrm{CI})$

Heterogeneity: $\mathrm{Tau}^{2}=0.00 ; \mathrm{Chi}^{2}=0.44, \mathrm{df}=1(\mathrm{P}=0.51) ; \mathrm{I}^{2}=0 \%$

Test for overall effect: $Z=0.98(P=0.32)$

\section{(b) RPQ-13}

Study or Subgroup Std. Mean Difference SE Weight

Moussavi Z. 2019

Rao V. 2019

Stilling J. 2020

Total $(95 \% \mathrm{Cl})$

Heterogeneity: $\mathrm{Tau}^{2}=0.00 ; \mathrm{Chi}^{2}=0.51, \mathrm{df}=2(\mathrm{P}=0.78) ; \mathrm{I}^{2}=0 \%$

Test for overall effect: $Z=2.12(P=0.03)$
$-0.10[-1.57,1.37]$

$0.46[-0.30,1.22]$

$0.34[-0.34,1.02]$

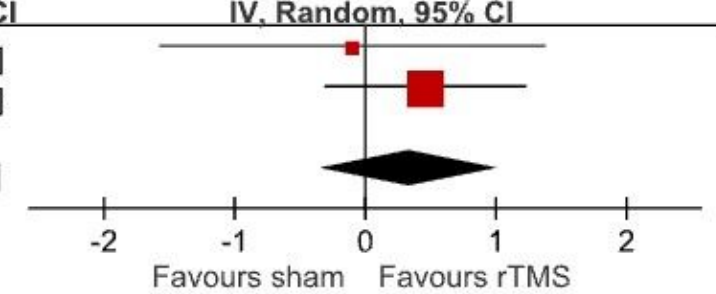

Std. Mean Difference IV. Random. $95 \% \mathrm{Cl}$

Std. Mean Difference IV. Random. $95 \% \mathrm{Cl}$

$0.70[-3.96,5.36]$

$-0.53[-1.00,-0.06]$

$0.48[-3.44,4.40]$

$-0.50[-0.97,-0.04]$

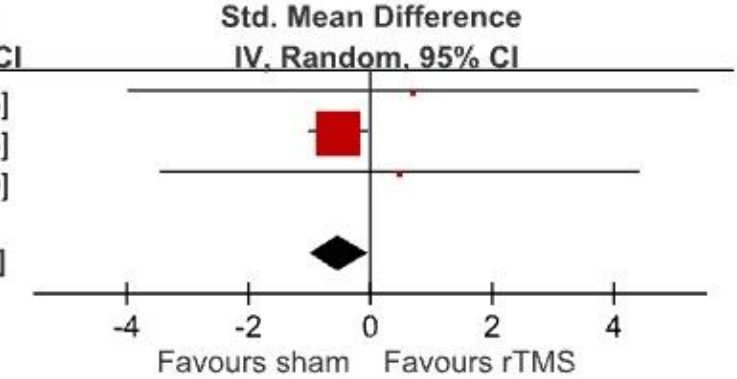

Figure 4

Forest plot showing efficacy of treatment versus sham treatment in postconcussion syndrome (a) RPQ-3 and (b) RPQ-13 